\title{
A CLINICO-MORPHOLOGIC STUDY OF MYELOPROLIFERATIVE NEOPLASMS
}

\author{
Lakshmi Rajagopal', Kavitha Ravi2, Santha Sadasivan ${ }^{3}$
}

1 Senior Resident, Department of Pathology, Government Medical College, Thiruvananthapuram.

${ }^{2}$ Associate Professor, Department of Pathology, Government Medical College, Thiruvananthapuram.

${ }^{3}$ Professor, Department of Pathology, Government Medical College, Thiruvananthapuram.

\section{ABSTRACT}

\section{BACKGROUND}

The concept of 'Myeloproliferative Neoplasms' (MPNs) was introduced by William Dameshek1 in 1951 by grouping together Chronic Myeloid Leukaemia (CML), Polycythaemia Rubra Vera (PRV), Essential Thrombocythaemia (ET), Primary Myelofibrosis (PMF) and Erythroleukaemia; a self-perpetuating trilineage myeloproliferation was underlined as the pathogenesis. JAK2 is one of the 4 Janus Kinases, which are non-receptor tyrosine kinases that are integral part of the cytokine and growth factor signalling. Its autoinhibitory function is released by a gain of mutation in the JAK2 V617F mutation. It leads to downstream activation of several signalling cascades like STAT proteins, PI3K-AKT pathway and mitogen activated protein kinases. Recent studies suggest that mutations in the TET2 tumour suppressor gene, which have been found in 17\% of JAK2 V617F+ Ph- MPNs and 7\% of JAK2V617Ecases, likely precede the acquisition of the JAK2 V617F mutation. Detection of mutation can be by Direct sequencing, Restriction fragment length polymorphism or allele-specific PCR. JAK2 Exon 12 Mutation is specific to JAK2 V617F negative PRV73. JAK2 N542E543 deletion is the most frequent among the ones described. These patients are usually heterozygous for the mutation with Erythroid myelopoiesis, subnormal serum erythropoietin levels and younger age at diagnosis. Apart from BCR-ABL mutation, others include JAK2 V617E, JAK2 EXON12, MPL, KIT, Calreticulin and TET2 mutations.

The Objectives of our Study were-

1. To study the clinico-morphologic features of Myeloproliferative Neoplasms with reference to the 2008 revised WHO classification of myeloid neoplasms.

2. To study the prevalence of JAK2 V617E in our region.

\section{MATERIALS AND METHODS}

It will be a descriptive study that is set in the Haematology Clinical Pathology Laboratory of MCH, Thiruvananthapuram. The bone marrow samples that are obtained from the Department of Medicine, which show features of MPNs are selected (based on the revised criteria). For these selected cases, the clinical history is collected and is compared with the peripheral smear, bone marrow aspirate, trephine and reticulin stain and the MPN is typed. JAK2 V617E study is advised for the cases of PRV.

\section{RESULTS}

A total of 60 Myeloproliferative Neoplasms were studied with a male predominance (63.2\%) and the most frequent age group being 40 - 80 years. Most were cases of Polycythaemia Rubra Vera (35\%) followed by Essential Thrombocythaemia (30\%), Chronic Myeloid Leukaemia and Progressive Myelofibrosis (15\%), Chronic Neutrophilic Leukaemia and Chronic Eosinophilic Leukaemia. Most cases were incidentally detected and a statistically significant relationship was noted between presenting complaint and the type of MPN The cases of MPN were studied for JAK2 mutation status as well, showed 24\% positivity with 9.5\% cases being lost to followup. Among the JAK2 positive cases, $60 \%$ presented with thrombotic episodes and $60 \%$ had splenomegaly.

\section{CONCLUSION}

60 cases of MPN were studied; cases selected in accordance with the 2008 WHO classification of myeloid neoplasms. Polycythaemia Rubra Vera is the commonest. Cases of PRV, ET, CIMF, CNL and CEL are being identified with more frequency compared to the earlier study conducted in our Department in 1994, probably because of increased awareness of these conditions and also better and clearcut diagnostic criteria available.

\section{KEYWORDS}

Myeloproliferative Neoplasms, Polycythaemia Rubra Vera, JAK2 V617E.

HOW TO CITE THIS ARTICLE: Rajagopal L, Ravi K, Sadasivan S. A clinico-morphologic study of myeloproliferative neoplasms. J. Evolution Med. Dent. Sci. 2017;6(17):1375-1378, DOI: 10.14260/Jemds/2017/299

Financial or Other, Competing Interest: None.

Submission 18-01-2017, Peer Review 11-02-2017,

Acceptance 17-02-2017, Published 27-02-2017.

Corresponding Author:

Dr. Lakshmi Rajagopal,

Lakshmi, NPP Road,

Oruvathilkotta

Anayara,

Thiruvananthapuram.

E-mail: laxmira4288gmail.com

DOI: $10.14260 /$ jemds $/ 2017 / 299$

(c) $(\mathrm{P})($

\section{BACKGROUND}

The concept of 'Myeloproliferative Neoplasms' (MPNs) was introduced by William Dameshek ${ }^{1}$ in 1951 by grouping together Chronic Myeloid Leukaemia (CML), Polycythaemia Rubra Vera (PRV), Essential Thrombocythaemia (ET), Primary Myelofibrosis (PMF) and Erythroleukaemia; a selfperpetuating trilineage myeloproliferation was underlined as the pathogenesis.

JAK2 is one of the 4 Janus Kinases which are non-receptor tyrosine kinases that are integral part of the cytokine and growth factor signalling. Its autoinhibitory function is released by a gain of mutation in the JAK2 V617F mutation. It leads to 
downstream activation of several signalling cascades like STAT proteins, PI3K-AKT pathway and mitogen-activated protein kinases. ${ }^{2,3,4}$

However, this lesion requires additional cooperating genetic aberrations and in fact is likely not the diseaseinitiating event. Although a 5- to 7-fold elevated risk of developing, an MPN among first-degree relatives of patients with MPN has been noted, nogermline JAK2 V617F mutations have been detected.

Recent studies suggest that mutations in the TET2 tumour suppressor gene, which have been found in $17 \%$ of JAK2 $\mathrm{V} 617 \mathrm{~F}+\mathrm{Ph}-\mathrm{MPNs}$ and $7 \%$ of JAK2 V617F - cases, likely precede the acquisition of the JAK2 V617F mutation.5,6,7

Detection of mutation can be by Direct sequencing, Restriction fragment length polymorphism or allele specific PCR.

\section{JAK2 Exon 12 Mutation}

It is specific to JAK2 V617F negative PRV73. JAK2 N542-E543 deletion is the most frequent among the ones described. These patients are usually heterozygous for the mutation with erythroid myelopoiesis, subnormal serum erythropoietin levels and younger age at diagnosis.8,9,10 Apart from BCR-ABL mutation, others include JAK2 V617E, JAK2 EXON12, MPL, KIT, Calreticulin and TET2 mutations.

\section{Objective}

1. To study the clinico-morphologic features of Myeloproliferative Neoplasms with reference to the 2008 revised WHO classification of myeloid neoplasms.

2. To study the prevalence of JAK2 V617E in our region.

\section{MATERIALS AND METHODS}

Descriptive study done in the Clinical Pathology Laboratory, Government Medical College, Thiruvananthapuram. Sixty cases with features of myeloproliferative neoplasm by Complete Blood Count, Peripheral smear and bone marrow findings were selected from the period of two years from August 2013 to August 2015.

\section{The following Data were Collected Based on the Proforma} Prepared

1. Haemoglobin.

2. Packed cell volume.

3. Total count.

4. Differential count.

5. Platelet count.

Clinical History and Physical examination findings were collected and the blood picture and bone marrow features were studied. In cases with features of PRV, JAK2 mutation status was studied.

The bone marrow specimens were processed and paraffin embedded and 5 micron thick sections were prepared. $\mathrm{H}$ and E stain and reticulin were done in all cases. Stains like Perl's were done in relevant cases. JAK2 mutation was done in the relevant cases of PRV.

\section{Classification of Myeloid Malignancies}

- Myeloproliferative Neoplasms (MPN). ${ }^{11}$

- Chronic myelogenous leukaemia, BCR-ABL1-positive.

- Chronic neutrophilic leukaemia.

- Polycythaemia vera.

- Primary myelofibrosis.

- Essential Thrombocythaemia.

- Chronic eosinophilic leukaemia, not otherwise specified.

- Mastocytosis.

- Myeloproliferative neoplasms, unclassifiable.

- Myelodysplastic Syndromes (MDS).

- Refractory cytopenia with unilineage dysplasia.

1. Refractory anaemia.

2. Refractory neutropenia.

3. Refractory thrombocytopenia.

- Refractory anaemia with ring sideroblasts.

- Refractory cytopenia with multilineage dysplasia.

- Refractory anaemia with excess blasts.

- Myelodysplastic syndrome with isolated del(5q).

- Myelodysplastic syndrome, unclassifiable.

- Childhood myelodysplastic syndrome.

- MDS/MPN.

- Chronic myelomonocytic leukaemia.

- Atypical chronic myeloid leukaemia, BCR-ABL1-negative.

- Juvenile myelomonocytic leukaemia.

- MDS/MPN, unclassifiable including refractory anaemia with ring sideroblasts and thrombocytosis.

- Myeloid and lymphoid neoplasms associated with eosinophilia and genetic abnormalties.8,9

- Myeloid and lymphoid neoplasms associated with PDGFRA rearrangement.

- Myeloid neoplasms associated with PDGFR-B rearrangement.

- Myeloid and lymphoid neoplasms associated with FGFR1 abnormalities.

- Acute Leukaemia.

2008 Classification gives the following Criteria for Diagnosis of PRV

Major Criteria12

1. Haemoglobin $>18.5 \mathrm{~g} / \mathrm{dL}$ (men) $>16.5 \mathrm{~g} / \mathrm{dL}$ (women).

2. Presence of JAK2 V617F or similar mutation.

\section{Minor Criteria}

1. Bone marrow trilineage myeloproliferation.

2. Subnormal serum erythropoietin level.

3. Endogenous erythroid colony growth.

Diagnosis of polycythaemia vera requires meeting either both major criteria and one minor criterion or the first major criterion and two minor criteria.

\section{RESULTS}

Out of the 60 cases of MPN studied, a male predominance (63.2\%) was noted with the most frequent age group being 40 - 80 years. Most were cases of PRV (35\%) followed by ET (30\%), CML and PMF (15\% each), Chronic Neutrophilic Leukaemia (1.7\%) and Chronic Eosinophilic Leukaemia (3.3\%). 


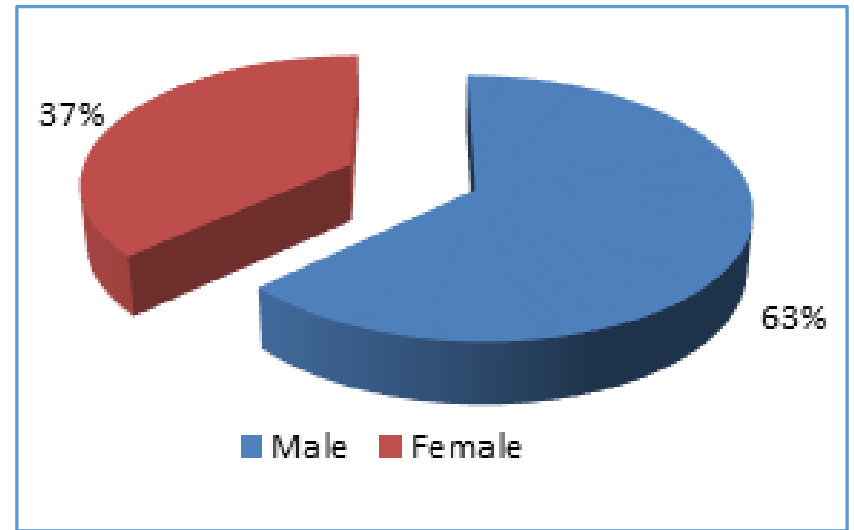

Figure 1. Pie-Chart of Percentage Distribution of Sample according to Sex

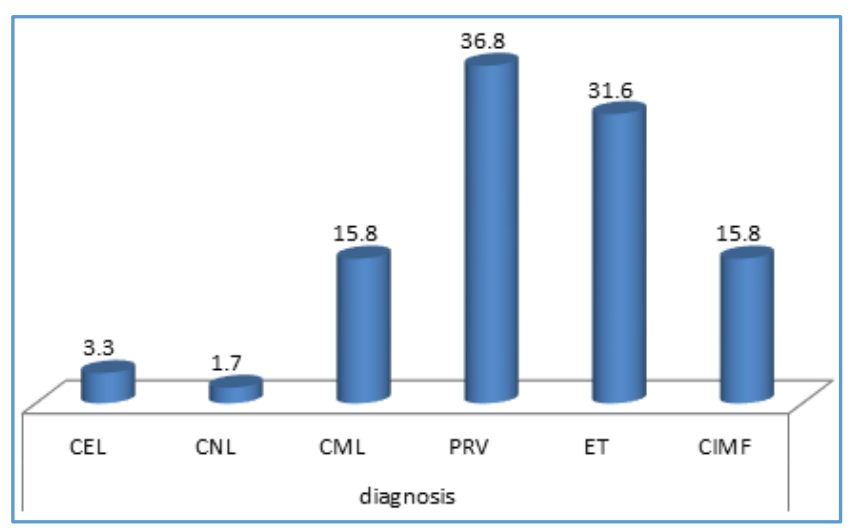

Figure 2. Percentage Distribution of Sample according to Diagnosis

Most cases were incidentally detected and a statistically significant relationship was noted between presenting complaint and the type of MPN. Thrombotic episodes as presenting complaint was mostly found in PRV.

\begin{tabular}{|c|c|c|c|c|c|c|}
\hline $\begin{array}{c}\text { Presenting } \\
\text { Complaint }\end{array}$ & CML & PRV & ET & CIMF & $x_{\mathbf{2}}$ & $\begin{array}{c}\text { P } \\
\text { value }\end{array}$ \\
\hline Thrombotic & $0(0)$ & $\begin{array}{c}6 \\
(66.7)\end{array}$ & $\begin{array}{c}3 \\
(33.3)\end{array}$ & $0(0)$ & & \\
\hline TIA & $0(0)$ & $\begin{array}{c}5 \\
(62.5)\end{array}$ & $\begin{array}{c}3 \\
(37.5)\end{array}$ & $0(0)$ & & \\
\hline $\begin{array}{c}\text { Incidentally } \\
\text { Detected }\end{array}$ & $\begin{array}{c}5 \\
(23.8)\end{array}$ & $\begin{array}{c}7 \\
(33.3)\end{array}$ & $\begin{array}{c}8 \\
(38.1)\end{array}$ & $\begin{array}{c}1 \\
(4.8)\end{array}$ & 23.51 & 0.005 \\
\hline Constitutional & $\begin{array}{c}4 \\
(21.1)\end{array}$ & $\begin{array}{c}3 \\
(15.8)\end{array}$ & $\begin{array}{c}4 \\
(21.1)\end{array}$ & $\begin{array}{c}8 \\
(42.1)\end{array}$ & & \\
\hline \multicolumn{7}{|c|}{ Table 1. Table showing Association of Presenting } \\
Complaint with Diagnosis \\
\hline
\end{tabular}

Amongst 21 cases of PRV a male preponderance was noted and JAK2 V617E analysis showed $24 \%$ positivity with $9.5 \%$ cases being lost to followup. Among the JAK2 positive cases, $60 \%$ presented with thrombotic episodes and $60 \%$ had splenomegaly.

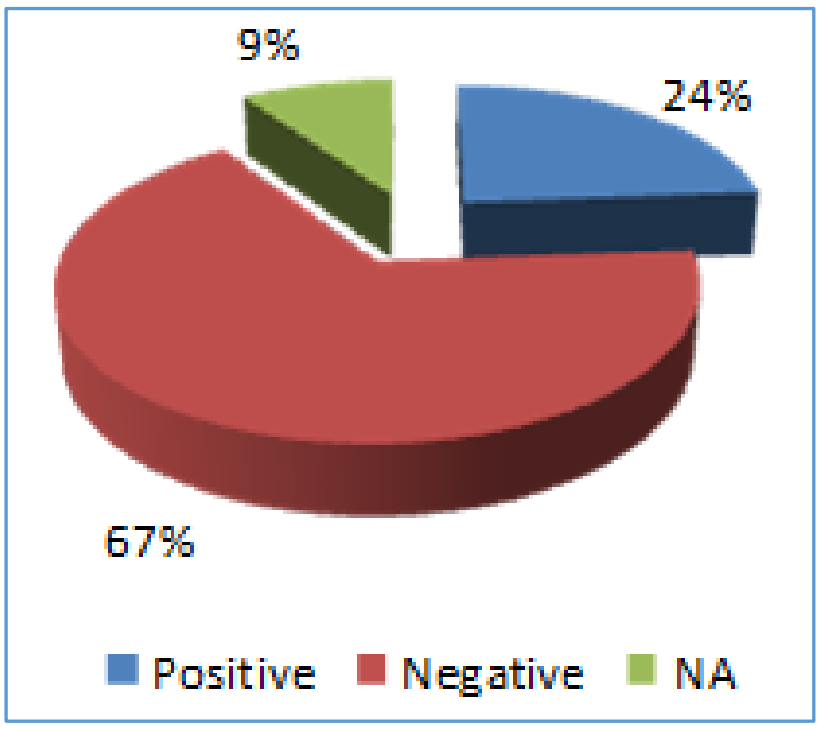

\section{Figure 3. Pie Chart showing Percentage Distribution of JAK2 Mutation in PRV}

\section{DISCUSSION}

Myeloproliferative neoplasm is a relatively rare entity studied in our clinical setting. Since its introduction in 1951, its diagnostic criteria has undergone a number of revisions with the most recent one during the period of $2005-2008$ with the discovery of the JAK2 mutations; namely JAK2 V617F, JAK2 exon 12 and the additional MPL, TET2 and calreticulin mutations.

In my study, 60 cases with peripheral blood and bone marrow evidence of trilineage proliferation were selected.

In my study, the maximum number of cases were PRV at $35 \%$ followed by ET at 30\% followed by PMF and CML. In addition, two cases of Chronic Eosinophilic Leukaemia and one case of chronic Neutrophilic Leukaemia were also obtained. In studies like Akram Ashgani et al, Sazawal et al and M. A. Sadiq et al done in Southeast Asian population and studied only the BCR-ABL negative MPNs, PRV and ET were the commonly encountered MPNs similar to our study.

\begin{tabular}{|c|c|c|c|}
\hline Study & PRV & ET & PMF \\
\hline M. A. Sadiq et al $^{13}$ & $35 \%$ & $39 \%$ & $26 \%$ \\
\hline Akram Asghani et al $14^{14}$ & $47.2 \%$ & $44 \%$ & $8.8 \%$ \\
\hline Sazawal et al ${ }^{15}$ & $45.3 \%$ & $13.3 \%$ & $41.4 \%$ \\
\hline \multicolumn{2}{|c|}{ Table 2. Table comparing Different } \\
Studies used as Reference \\
\hline
\end{tabular}

The mean age in all the studies are in agreement.

\begin{tabular}{|c|c|}
\hline Study & Age \\
\hline M. A. Sadiq et al ${ }^{13}$ & $54+/-15^{*}, 41+/-14^{* *}$ \\
\hline Akram Ashgani et al ${ }^{14}$ & $51+/-10$ \\
\hline Sazawa et al ${ }^{15}$ & $53+/-11$ \\
\hline
\end{tabular}

Except in a study conducted at Tehran, Iran, a male predominance was noted. But it could probably be because of the under representation of females. Fewer number of females are seeking medical attention and this problem needs to be addressed. 


\begin{tabular}{|c|c|}
\hline Study & Male: Female Ratio \\
\hline${\text { M. A. Sadiq et } \text { al }^{13}}$. $^{14}$ & $1.5: 1$ \\
\hline Akram Ashgari et al $^{14}$ & $0.7: 1$ \\
\hline Sazawal et al ${ }^{15}$ & $2.8: 1$ \\
\hline
\end{tabular}

In our study, most cases were incidentally detected followed by those with constitutional symptoms, then TIA and thrombotic episodes. On comparing the presenting complaint of different MPNs, the p-value was found to be 0.005 which is clinically significant. Hence, MPNs like PRV and ET have greater propensity for presenting with thrombotic episodes when compared to the other MPNs. Sazawal et al and Popov et al found more thrombotic risk in JAK2 V617F positive MPN. Since PRV and ET have greater chance for the same, we can infer that PRV and ET have more thrombotic risk which appears to be increasing with the counts.

Regarding past history, the p-value of this association was found to be 0.053 , which is not statistically significant. Hence, it can be inferred that past history does not have much bearing on the diagnosis.

We got only 21 cases of PRV in our study period; it was compared with a study conducted by Tefferi et al in 2011. In our study, the cases had a median age of 53 years $(29 \%$ below 40).

- Sex ratio was 2:1.

- Vasomotor symptoms were not seen in any.

- 6 out of 21 cases had presented with a thrombotic episode (29\%).

- Hepatosplenomegaly was seen in $62 \%$ of cases.

- All had subnormal serum erythropoietin.

- Hb level was $>/=18.5$ in two-third of the cases.

- Out of the 21 cases, $24 \%$ showed JAK2 V617F positivity, 2 cases were lost to followup (9\%).

My study was conducted in a period of 18 months, hence conducting a survival or life expectancy study is not feasible. Similarly, rate of blastic transformation or development of marrow fibrosis also takes considerable time and is not practical in an 18-month study. In our setting once a diagnosis of PRV is made by bone marrow examination, JAK2 analysis is not done as a routine test because of its cost. These patients are managed with phlebotomy bi-yearly and a course of hydroxyurea is given. The effectiveness of tyrosine kinase inhibitors in these patients is still under investigation.

The limitation of my study is that it is a descriptive study done for a period of 18 months; hence, fewer number of cases are studied and survival analysis is not possible. Also, only JAK2 V617F could be studied; other mutational analysis like JAK2 exon 12, TET2, MPL and Calreticulin are not available.

\section{CONCLUSION}

Since the introduction of the 2008 WHO classification of myeloid neoplasms, there has been a better diagnostic criterion for MPN. There has been increased rate of detection of entities of entities like ET, PRV, CNL and CEL owing to clearcut diagnostic criteria and molecular findings.

In cases of PRV, there is greater propensity to present with thrombotic episodes and a JAK2 V617F positivity of $24 \%$. It could be used for targeted therapy depending on the affordability.

\section{REFERENCES}

[1] Dameshek W. Some speculations on the myeloproliferative syndromes. Blood 1951;6(4):3725.

[2] Jamieson $\mathrm{CH}$, Gotlib J, Durocher JA, et al. The JAK2 V617F mutation occurs in hematopoietic stem cells in polycythemia vera and predisposes toward erythroid differentiation. Proc Natl Acad Sci U S A 2006;103(16):6224-9.

[3] Khwaja A. The role of Janus kinases in haemopoiesis and haematological malignancy. $\mathrm{Br} \mathrm{J}$ Haematol 2006;134(4):366-84.

[4] Delhommeau F, Dupont S, Dela Valle V, et al. Mutation in TET2 in myeloid cancers. $N$ Engl J Med 2009;360(22):2289-301.

[5] Tefferi A, Pardanani A, Lim KH, et al. TET2 mutations and their clinical correlates in polycythemia vera, essential thrombocythemia and myelofibrosis. Leukemia 2009;23(5):905-11.

[6] Jankowska AM, Szpurka H, Tiu RV, et al. Loss of heterozygosity 4q24 and TET2 mutations associated with myelodysplastic/myeloproliferative neoplasms. Blood 2009;113(25):6403-10.

[7] Delhommeau F, Dupont S, Tonetti C, et al. Evidence that the JAK2 G1849T (V617F) mutation occurs in a lymphomyeloid progenitor in polycythemiavera and idiopathic myelofibrosis. Blood 2007;109(1):71-7.

[8] Pardanani A, Lasho TL, Finke C, et al. Prevalence and clinicopathological correlates of JAK2 exon 12 mutations in JAK2V617E negative polycythemia vera. Leukemia 2007;21(9):1960-3.

[9] Pietra D, Li S, Brisci A, et al. Somatic mutations of JAK2 exon 12 in patients with JAK2(V617F)-negative myeloproliferative disorders. Blood 2008;111(3):1686-9.

[10] Passamonti F, Schnittger S, Girdon F, et al. Molecular and clinical features of myeloproliferative neoplasm associated with JAK2 exon 12 mutations: a European multicentre study. Blood 2009;114:3904.

[11] Găman M, Vlădăreanu $\mathrm{AM}$, Radesi $\mathrm{S}$, et al. The implications of revised WHO classification (2008) of chronic myeloid neoplasms. Rom J Intern Med 2011;49(1):25-30.

[12] Vardiman JW, Thiele J, Arber DA, et al. The 2008 revision of the WHO classification of myeloid neoplasms and acute leukemia: rationale and important changes. Blood 2009;114(5):937-51.

[13] Sadiq MA, Ahmed S, Ali N. Frequency of janus associated kinase 2 (JAK2) mutation in patients of bcrabl negative myeloproliferative neoplasms. Applied Life Sciences 2013;63(2):235-40.

[14] Asghari A, Ahmadi AS, Basi A, et al. The association between prevalence of JAK2V617E mutation and blood indicies in groups of patients with myeloproliferative neoplasms in Rasul Akram hospital. International Journal of Hematology-Oncology and Stem Cell Research 2011;5(4):10-3.

[15] Sazawal S, Bajaj J, Chikkara S, et al. Prevalence of JAK2 V617F mutation in Indian patients with chronic myeloproliferative disorders. Indian J Med Res 2010;132:423-7. 